\title{
Increased Risk of Surgical-Site Infection and Need for Manipulation Under Anesthesia for Those Who Undergo Open Versus Arthroscopic Rotator Cuff Repair
}

\author{
Kevin Y. Wang, B.A., Amil R. Agarwal, B.A., Amy L. Xu, B.S., Matthew J. Best, M.D., \\ R. Timothy Kreulen, M.D., Meghana Jami, B.S., Edward G. McFarland, M.D., and \\ Uma Srikumaran, M.D., M.B.A., M.P.H.
}

\begin{abstract}
Purpose: To compare 90-day postoperative complications, health care use, 2-year and 5-year rates of reoperation and manipulation under anesthesia, and costs at the 30-day, 90-day, and 1-year postoperative intervals following open and arthroscopic rotator cuff repair (RCR). Methods: Patients who underwent an open or arthroscopic RCR with minimum 5-year follow-up were identified in a national database (PearlDiver Technologies) using Common Procedural Terminology and International Classification of Diseases codes. These patients were then stratified into 2 cohorts: open RCRs and arthroscopic RCRs. These cohorts were propensity-matched based on age, sex, Charlson Comorbidity Index, smoking status, and obesity (body mass index>30). 90-day medical complications, 2-year and 5-year surgical complications, and reimbursements at the 30-day, 90-day and 1-year postoperative intervals were assessed. Bivariate statistics were performed using $\chi^{2}$ tests, Fisher exact tests, and Student $t$ tests where appropriate. Reimbursements included the reimbursement for the index surgery as well as any reimbursements during the specified postoperative interval related to the index surgery. Results: In total, 3,266 patients who underwent open RCR were matched with 3,266 patients who underwent arthroscopic RCR. Compared with patients who underwent arthroscopic RCR, patients who underwent open RCR were at significantly increased risk of 90-day surgical-site infection $(0.89 \%$ vs $0.34 \%, P=.004)$, undergoing manipulation under anesthesia (MUA) within 2 years of surgery (1.65\% vs $0.95 \%, P=.012)$, and undergoing MUA within 5 years of surgery $(1.75 \%$ vs $1.10 \%, P=.028)$. There were no significant differences in any other postoperative complications, reoperation rates, or reimbursements between open RCR and arthroscopic RCR (all, $P>$.05). Conclusions: Patients undergoing open RCR were at increased risk of 90-day surgical-site infection and MUA both within 2 years and within 5 years of surgery in this study cohort. Level of Evidence: Level III, retrospective cohort study.
\end{abstract}

D egenerative rotator cuff pathology is the most common cause of shoulder disability. Surgical repair of rotator cuff injury can be performed with either arthroscopic or open techniques, with recent trends favoring the use of arthroscopic rotator cuff

From the Department of Orthopaedic Surgery, Johns Hopkins, Columbia, Maryland (K.Y.W., A.L.X., R.T.K., M.J., E.G.M., U.S.); Department of Orthopaedic Surgery, George Washington School of Medicine and Health Sciences, Washington DC (A.R.A.); and Department of Orthopaedic Surgery, Massachusetts General Hospital, Boston, Massachusetts (M.J.B.), U.S.A.

The authors report the following potential conflicts of interest or sources of funding: U.S. reports personal fees and other from Tigon Medical; personal fees from Conventus, Fx Shoulder USA, and Orthofix; grants from DePuy Synthes, Arthrex, Wright, and Smith $\theta$ Nephew; personal fees from Heron and Pacira; and grants from ASES and OMEGA, outside the submitted work. In addition, he has a patent from Conventus pending, a patent from $F x$ Shoulder USA pending, and a patent from Tigon Medical issued. Full ICMJE repair (RCR) over open RCR. ${ }^{1}$ A 2019 study reported that the rate of arthroscopic RCR increased from $73 \%$ in 2007 to $90 \%$ in 2017 , whereas the rate of open or mini-open RCR decreased from $27 \%$ to $10 \%$ during the same period. ${ }^{1}$ Potential advantages to the arthroscopic

author disclosure forms are available for this article online, as supplementary material.

Received March 22, 2021; accepted November 13, 2021.

Address correspondence to Uma Srikumaran, M.D., M.B.A., M.P.H., Department of Orthopaedic Surgery, Adult Reconstruction Division, Johns Hopkins, 10700 CharterDr., Suite 205,Columbia, MD 21044.E-mail:us@ jhmi.edu

(C) 2021 THE AUTHORS. Published by Elsevier Inc. on behalf of the Arthroscopy Association of North America. This is an open access article under the CC BY-NC-ND license (http://creativecommons.org/licenses/by-nc-nd/4.0/). 2666-061X/21381

https://doi.org/10.1016/j.asmr.2021.11.012 
approach include lower risk of deltoid detachment, smaller skin incisions, less soft-tissue dissection, and greater capability for treating intra-articular lesions..$^{2-4}$ However, some surgeons still prefer open repair since the open approach may allow for placement of stronger stitches and easier transosseous fixation for reproducible restoration of the supraspinatus footprint. ${ }^{5-7}$ In addition, more experience is required to become proficient in arthroscopic techniques, which may be difficult for some surgeons operating at low-volume centers. $^{8}$

Existing literature comparing functional outcomes and complication rates between arthroscopic RCR and open RCR presents mixed conclusions. A 2008 metaanalysis found no significant difference in postoperative complications or functional outcome scores between patients who underwent arthroscopic RCR and mini-open repair. ${ }^{9}$ A subsequent 2010 systematic review reported no statistically significant difference in postoperative American Shoulder and Elbow Surgeons score, University of California Los Angeles shoulder score, various pain scores, or rate of recurrent rotator cuff tears between arthroscopic and mini-open RCR repairs. ${ }^{10}$ More recent studies, however, have reported lower rates of reoperation and overall complications following arthroscopic RCR compared with open RCR, perhaps due to surgeons gaining more experience with arthroscopic techniques. ${ }^{1,11}$ However, these previous investigations on outcomes and complications following open versus arthroscopic RCR are mostly limited to 2-year follow-up, and more evidence regarding long-term postoperative complications is needed. Further, the procedural equipment and longer operative times for arthroscopic RCRs reported in some series have been associated with greater costs, but more data are needed to determine differences in short and long-term cost effectiveness between open and arthroscopic techniques. ${ }^{12-15}$

The purpose of this study was to compare 90-day postoperative complications, health care use, 2-year and 5-year rates of reoperation and manipulation under anesthesia, and costs at the 30-day, 90-day, and 1year postoperative intervals following open and arthroscopic RCR. It was hypothesized that, compared with open RCR, arthroscopic RCR would have lower 90-day postoperative complication rates but greater reimbursements at all postoperative time intervals.

\section{Methods}

This study was deemed exempt from institutional review board approval. A retrospective cohort analysis was conducted using administrative claim's data acquired from 2010 to 2018 . The data were collected from the Mariner subset of the PearlDiver Patient Records Database, Colorado Springs, CO; Www.pearldiverinc. com). The Mariner subset includes all payer's claims data from more than 121 million patients and longitudinally tracks these patients using a distinct all patient identifier. From the 121 million patients in the Mariner subset, the MOrtho dataset was used, which contains a randomized sample of 15 million patients. Patients who underwent an open (Current Procedural Terminology [CPT]-23412, CPT-23410) or arthroscopic (CPT-29827) primary RCR for the first time were separately identified using CPT codes. Primary RCR was able to be determined using the "first instance" feature provided by the PearlDiver database for each procedure. Patients were included if they had at least 5-year follow-up. Since 5-year follow-up was required, only patients who underwent total shoulder arthroplasty from 2010 to 2013 were included in this study. Patients with any previous cuff surgery were excluded from either group. A flowchart diagramming our inclusion and exclusion criteria is displayed in Figure 1.

\section{Demographics and Outcomes}

Patient demographic characteristics collected include age, sex, Charlson Comorbidity Index (CCI), obesity (body mass index $>30$ ), and smoking status. The primary outcomes for this study were 90-day medical complications, rates of 2- and 5-year reoperation, and manipulation under anesthesia (MUA). Any subsequent open or arthroscopic shoulder surgery, including shoulder arthroplasty, after the primary RCR was considered a reoperation. Ninety-day medical complications assessed included surgical-site infection, postoperative renal failure, postoperative anemia, atrial fibrillation, arrhythmia without atrial fibrillation, bleeding complications, blood transfusion, pneumonia, stroke, death, deep-vein thrombosis, pulmonary embolism, heart failure, respiratory failure, and sepsis. Secondary outcomes for this study included 90-day readmissions rates as well as 30-day, 90-day, and 1year reimbursements.

\section{Propensity Score Matching}

Propensity-matched cohorts of patients who had an open RCR and those who had an arthroscopic RCR were created to control for measured covariates and mitigate potential confounders. The propensity score was defined as the conditional probability of having undergone an open or arthroscopic RCR based on age, CCI, obesity status, and smoking status. Matching was conducted using a $1: 1$ nearest neighbor matching ratio by univariate analysis. This was done using demographics collected at the time of RCR among the open and arthroscopic RCR cohorts. Propensity score matching was conducted using R software provided by PearlDiver.

\section{Statistical Analysis}

Data on patient demographics, complications, and reimbursements were analyzed between the matched 




Fig 1. Inclusion and exclusion criteria. (MOrtho, dataset used in Mariner subset of PearlDiver; RCR, rotator cuff repair.)

cohorts with univariate analysis using $\mathrm{R}$ software provided by PearlDiver. Univariate analysis was conducted using $\chi^{2}$ tests or Student $t$ tests where appropriate. Statistical comparisons were conducted using the group of interest against the control group. A $P$ value of $<.05$ was used as the cutoff for significance for univariate analysis.

\section{Results}

In total, 3,266 patients undergoing open RCR were matched with 3,266 patients undergoing arthroscopic RCR. There were no significant differences between the matched cohorts in terms of patient demographics or comorbidities (Table 1).

\section{0-Day Postoperative Complications, Emergency Department (ED) Visits, and Readmissions}

Compared with matched patients undergoing arthroscopic RCR, those undergoing open RCR had increased risk of 90 -day surgical-site infection $(0.89 \%$ vs $0.34 \%, P=.004$; Table 2 ). There were no significant differences between the 2 cohorts in terms of other 90 day complications, 90-day ED visits, or 90-day readmissions $(P>.05$ for all; Table 2$)$.

\section{2-Year and 5-Year Surgical Complications}

Compared with matched patients undergoing arthroscopic RCR, those undergoing open RCR had significantly greater rates of 2 -year MUA $(1.65 \%$ vs $0.95 \%, P=.012$; Table 3$)$ and 5 -year MUA ( $1.75 \%$ vs $1.10 \%, P=.028$; Table 3 ). There were no significant differences in 2 -year or 5 -year reoperation surgery rates between the 2 cohorts $(P>.05$ for all, Table 3$)$.

\section{Reimbursement}

There were no differences in reimbursements at the 30-day, 90-day, or 1-year postoperative intervals between the matched arthroscopic and open RCR cohorts $(P>.05$ for all, Table 4$)$.

\section{Discussion}

In the present study, patients who underwent open RCR were at increased risk of 90-day surgical-site infection as well as 2-year and 5-year MUA relative to patients who underwent arthroscopic RCR. There were

Table 1. Demographic Information for Open RCR and Arthroscopic RCR

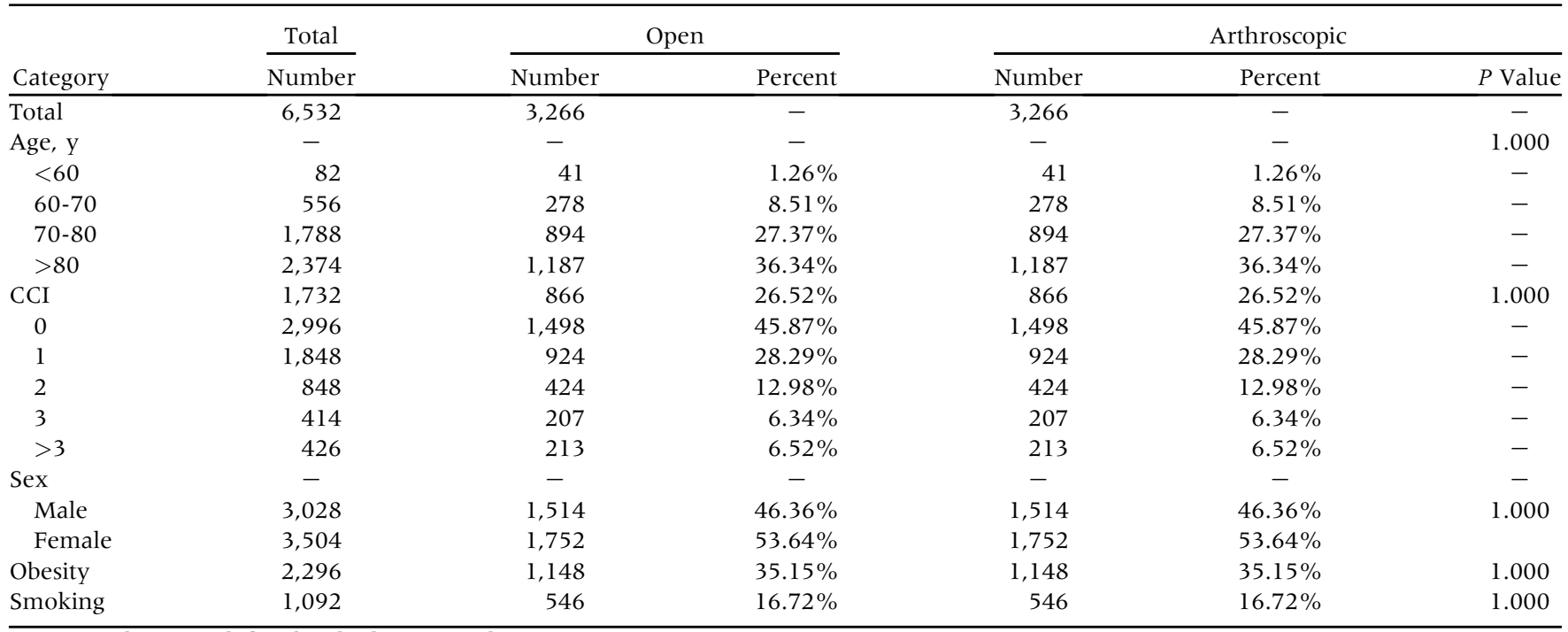

NOTE. Obesity is defined as body mass index $>30$.

CCI, Charlson Comorbidity Index; RCR, rotator cuff repair. 
Table 2. 90-Day Postoperative Complications, Readmissions, and ED Visits for Open RCR and Arthroscopic RCR

\begin{tabular}{|c|c|c|c|c|c|c|}
\hline Category & $\frac{\text { Total }}{\text { Number }}$ & \multicolumn{2}{|c|}{ Open } & \multicolumn{3}{|c|}{ Arthroscopic } \\
\hline & 6,532 & 3,266 & - & 3,266 & - & - \\
\hline ED visit & 452 & 234 & $7.16 \%$ & 218 & $6.67 \%$ & .435 \\
\hline SSI & 40 & 29 & $0.89 \%$ & 11 & $0.34 \%$ & .004 \\
\hline Renal failure & 30 & 18 & $0.55 \%$ & 12 & $0.37 \%$ & .272 \\
\hline Anemia & - & 16 & $0.49 \%$ & $<11$ & - & .161 \\
\hline Bleeding complication & - & $<11$ & - & $<11$ & - & .796 \\
\hline Blood transfusion & - & 13 & $0.40 \%$ & $<11$ & $0.28 \%$ & .393 \\
\hline Pneumonia & 63 & 29 & $0.89 \%$ & 34 & $1.04 \%$ & .527 \\
\hline Stroke & 44 & 22 & $0.67 \%$ & 22 & $0.67 \%$ & 1.000 \\
\hline Death & - & $<11$ & - & $<11$ & - & 1.000 \\
\hline DVT & 49 & 28 & $0.86 \%$ & 21 & $0.64 \%$ & .315 \\
\hline Sepsis & - & 13 & $0.40 \%$ & $<11$ & - & .393 \\
\hline
\end{tabular}

NOTE. Values in bold are statistically significant at $P<.05 ;<11$ : PearlDiver does not report values less than 11.

afib, atrial fibrillation; DVT, deep vein thrombosis; ED, emergency department; RCR, rotator cuff repair; SSI, surgical-site infection; UTI, urinary tract infection; w/, with; w/o, without.

no significant differences in ED visits, readmissions, reoperation rates, or reimbursements between arthroscopic and open RCR at any of the postoperative time intervals assessed.

This study's results align with previous literature reporting lower risk of infection rates for arthroscopic RCR compared with open RCR. ${ }^{1,16-19}$ In a singleinstitution retrospective study of 1,556 patients, Hughes et al. ${ }^{17}$ reported an increased postoperative infection rate following open RCR $(2.45 \%)$ compared with arthroscopic RCR $(0.44 \%)$. In a separate retrospective study of 1,824 patients, Vopat et al. ${ }^{19}$ further reported patients undergoing open/open-mini RCR had 8.63-fold greater odds of postoperative infection compared with arthroscopic RCR. It is hypothesized that the smaller incision needed for arthroscopic procedures compared to open procedures may contribute to these differences in infection rates found in the studies of Hughes et al. ${ }^{17}$ and Vopat et al., ${ }^{19}$ as well as in our study. Notably, this study did not find a significant difference in the rates of any other medical complications assessed between the 2 groups. There also were no differences in reoperation, readmission, or ED visitation rates, which suggests that the infections were able to be treated nonoperatively on an outpatient basis. This finding is supported by the results of previous studies which demonstrated only isolated increase in risk of postoperative infection but not medical complications following open RCR compared to arthroscopic RCR. ${ }^{11,20,21}$

Postoperative stiffness has been reported to be the most common surgical complication following RCR, with an incidence rate ranging from $2.7 \%$ to $15 \% .^{21-25}$ Since there is no International Classification of Diseases code for arthrofibrosis, MUA was assessed as a measure of severe postoperative shoulder stiffness requiring operative management, demonstrating that open RCR was associated with significantly greater incidence of MUA within 2 years and 5 years of surgery, compared with arthroscopic RCR. Early passive range of motion

Table 3. 2-Year and 5-Year Surgical Outcomes for Open RCR and Arthroscopic RCR

\begin{tabular}{|c|c|c|c|c|c|c|}
\hline Category & $\frac{\text { Total }}{\text { Number }}$ & \multicolumn{2}{|c|}{ Open } & \multicolumn{3}{|c|}{ Arthroscopic } \\
\hline 2-year reoperation & 771 & 391 & $11.97 \%$ & 380 & $11.64 \%$ & .673 \\
\hline 5 -year reoperation & 1122 & 559 & $17.12 \%$ & 563 & $17.24 \%$ & .896 \\
\hline 5-year MUA & 93 & 57 & $1.75 \%$ & 36 & $1.10 \%$ & .028 \\
\hline
\end{tabular}

NOTE. Values in bold are statistically significant at $P<.05$.

MUA, manipulation under anesthesia; RCR, rotator cuff repair. 
Table 4. 30-Day, 90-Day, and 1-Year Reimbursements for Open RCR and Arthroscopic RCR

\begin{tabular}{lcccc}
\hline & \multirow{2}{*}{ Open } & & \multicolumn{2}{c}{ Arthroscopic } \\
\cline { 2 - 2 } \cline { 5 - 5 } Category & Number & & Number & $P$ Value \\
\hline 30-day reimbursements & $\$ 2,280.96$ & & $\$ 2,225.35$ & .495 \\
90-day reimbursements & $\$ 3,420.78$ & & $\$ 3,419.10$ & .804 \\
1-year reimbursements & $\$ 9,117.81$ & & $\$ 8,931.72$ & .537 \\
\hline RCR, rotator cuff repair. & & &
\end{tabular}

following RCR is thought to decrease postoperative stiffness, and Walton et al. found that arthroscopic RCR led to greater passive range of motion at 3 months' and 6 months' postoperatively compared with open RCR. ${ }^{20,26}$ Interestingly, patient perceptions of stiffness were similar between the 2 groups in the study by Walton et al., even with the objective differences in range of motion, which further merits the use of MUA as a more objective measure of stiffness compared to solely patient perception. ${ }^{27}$

One hypothesis for the increased rates of MUA in the open cohort relative to arthroscopic cohort is that the greater invasiveness of open surgery mediates scar tissue formation that manifests clinically as joint stiffness. Anatomically, the more invasive nature of mini-open approaches requires separation of deltoid fibers extending into the subdeltoid bursa, increasing the possibility of developing postoperative subdeltoid adhesions and subsequent joint stiffness. ${ }^{28-31}$ In a retrospective study of 64 RCRs performed with an average of 45 months of follow-up, Severud et al. ${ }^{32}$ reported that $0 \%(0 / 35)$ of patients in the allarthroscopic cohort developed fibrous ankylosis, compared with $14 \%(4 / 29)$ in the mini-open cohort. Severud et al. ${ }^{32}$ also demonstrated significantly faster return to motion following arthroscopic RCR compared with mini-open repair. Currently, there are limited long-term data on postoperative stiffness and subsequent MUA following RCR, and the present study reporting surgical complications at 5 years after surgery contains the longest follow-up on this topic. While previous research with shorter follow-up periods have demonstrated increased stiffness and reduced range of motion following open RCR compared with arthroscopic RCR, the present study demonstrates that patients undergoing open RCR have increased rates of MUA up to 5 years after surgery.

Previous literature has shown that physical therapy can reduce postoperative stiffness and subsequent MUA in patients undergoing both open and arthroscopic RCR. ${ }^{27}$ However, the database used in this study was unable to determine the timing, type, and effectiveness of physical therapy after either open or arthroscopic RCR. As such, the results do not account for any aspects of physical therapy or other forms of postoperative rehabilitation.
In addition, this study demonstrated no significant difference in reoperation rates between open and arthroscopic RCR at the 2-year or 5-year postoperative intervals. Baker and $\mathrm{Liu}^{2}$ examined the National Surgical Quality Improvement Program database and found a significantly greater risk of returning to the operating room within 30 days of surgery for patients undergoing open RCR $(0.70 \%)$ compared with arthroscopic RCR $(0.26 \%)$. However, their study included any return to the operating room and did not specify that it was for shoulder surgery. Other studies in the literature support no difference in reoperation rates at 1- or 2-year follow up. For instance, Bishop et al. ${ }^{32}$ found no significant difference in reoperation rates following arthroscopic (2/32) and open (1/40) RCR within a year of index RCR. Carr et al. ${ }^{13}$ also reported equivalent reoperation rates between arthroscopic (2/136) and open (2/137) RCR at 2-year follow-up. The present study demonstrates that this equivalence in reoperation rates was maintained up to 5 years after the index procedure; notably, reoperations in this study was defined as all subsequent shoulder surgeries, including arthroplasty.

In terms of health care costs from the payor's perspective, this study found that there were no significant differences in reimbursements between open RCR and arthroscopic RCR at 30-day, 90-day, and 1 -year postoperative intervals. This is in contrast to a 2010 study by Adla et al., ${ }^{12}$ which found open RCR to be slightly more cost-effective than arthroscopic RCR at 1 -year postoperation, given the greater cost of materials used in arthroscopic RCR such as anchors, disposable cannulae, debridement tools, and other surgical equipment. Despite this initial greater cost of arthroscopic RCR, a 2015 randomized controlled trial by Carr et al. ${ }^{13}$ found that the cost-effectiveness at 2 years between arthroscopic and open RCR for degenerative rotator cuff tear in patients older than 50 years was not significantly different. It is important to note that these studies, including this one, encompass both surgical and postoperative costs.

\section{Limitations}

The results of this study should be interpreted in context of its limitations. Despite rigorous matching, the PearlDiver database lacks sufficient granularity to fully account for differences in whether there were concomitant procedures performed, the precise surgical indication, rotator cuff tear severity, and chronic versus acute tear. In addition, surgeon experience and personal preference were unable to be determined with the database. However, this study was able to control for other demographic variables and comorbidities that have been shown to alter postoperative recovery following RCR, such as age, obesity, and tobacco use. ${ }^{3,34}$ Other known risk factors were controlled for in 
the CCI index. However, we were unable to match specific comorbidities that may have a direct impact in outcomes, such as diabetes mellitus. This study also was not able to determine whether each RCR performed was a revision of a repair previously performed, or if it was the first RCR done for each patient. Furthermore, it is important to acknowledge that although the differences in rates of 90-day surgical-site infection, as well as 2-year and 5-year MUA between patients undergoing open RCR and arthroscopic RCR were statistically significant, they are not necessarily clinically significant. Finally, this study was conducted using a retrospective insurance claims database and is subject to potential biases inherent to limitations of administrative databases, such as ICD coding errors.

\section{Conclusions}

Patients undergoing open RCR were at increased risk of 90-day surgical-site infection as well as MUA both within 2 years and within 5 years of surgery in this study cohort.

\section{References}

1. Kelly BC, Constantinescu DS, Vap AR. Arthroscopic and open or mini-open rotator cuff repair trends and complication rates among American Board of Orthopaedic Surgeons Part II Examinees (2007-2017). Arthroscopy 2019;35:3019-3024.

2. Baker CL, Liu SH. Comparison of open and arthroscopically assisted rotator cuff repairs. Am J Sports Med 1995;23: 99- 104.

3. Levy HJ, Uribe JW, Delaney LG. Arthroscopic assisted rotator cuff repair: Preliminary results. Arthroscopy 1990;6: 55-60.

4. Paulos LE, Kody MH. Arthroscopically enhanced "miniapproach" to rotator cuff repair. Am J Sports Med 1994;22:19-25.

5. Apreleva M, Ozbaydar M, Fitzgibbons PG, Warner JJ. Rotator cuff tears: The effect of the reconstruction method on three-dimensional repair site area. Arthroscopy 2002;18:519-526.

6. Gerber C, Schneeberger AG, Beck M, Schlegel U. Mechanical strength of repairs of the rotator cuff. J Bone Joint Surg $\mathrm{Br}$ 1994;76:371-380.

7. Gerber C, Schneeberger AG, Perren SM, Nyffeler RW. Experimental rotator cuff repair. A preliminary study. J Bone Joint Surg Am 1999;81:1281-1290.

8. Yamaguchi K, Levine WN, Marra G, Galatz LM, Klepps S, Flatow EL. Transitioning to arthroscopic rotator cuff repair: The pros and cons. Instr Course Lect 2003;52:81-92 [review].

9. Morse K, Davis AD, Afra R, Kaye EK, Schepsis A, Voloshin I. Arthroscopic versus mini-open rotator cuff repair: A comprehensive review and meta-analysis. Am J Sports Med 2008;36:1824-1828.

10. Lindley K, Jones GL. Outcomes of arthroscopic versus open rotator cuff repair: A systematic review of the literature. Am J Orthop (Belle Mead, NJ) 2010;39:592-600 [review].

11. Day M, Westermann R, Duchman K, et al. Comparison of short-term complications after rotator cuff repair: Open versus arthroscopic. Arthroscopy 2018;34:1130-1136. doi: 10.1016/j.arthro.2017.10.027.

12. Adla DN, Rowsell M, Pandey R. Cost-effectiveness of open versus arthroscopic rotator cuff repair. J Shoulder Elbow Surg 2010;19:258-261.

13. Carr AJ, Cooper CD, Campbell MK, et al. Clinical effectiveness and cost-effectiveness of open and arthroscopic rotator cuff repair [the UK Rotator Cuff Surgery (UKUFF) randomised trial]. Health Technol Assess 2015;19:1-218.

14. Churchill RS, Ghorai JK. Total cost and operating room time comparison of rotator cuff repair techniques at low, intermediate, and high volume centers: Mini-open versus all-arthroscopic. J Shoulder Elbow Surg 2010;19:716-721.

15. Nicholson JA, Searle HKC, MacDonald D, McBirnie J. Cost-effectiveness and satisfaction following arthroscopic rotator cuff repair: Does age matter? Bone Joint J 2019;101-B:860-866.

16. Athwal GS, Sperling JW, Rispoli DM, Cofield RH. Deep infection after rotator cuff repair. J Shoulder Elbow Surg 2007;16:306-311.

17. Hughes JD, Hughes JL, Bartley JH, Hamilton WP, Brennan KL. Infection rates in arthroscopic versus open rotator cuff repair. Orthop J Sports Med 2017;5: 2325967117715416.

18. Osti L, Papalia R, Del Buono A, Denaro V, Maffulli N. Understanding and preventing complications in repairing rotator cuff tears. Med Sport Sci 2012;57:178-183. doi:10. $1159 / 000329812$ [review].

19. Vopat BG, Lee BJ, DeStefano S, et al. Risk factors for infection after rotator cuff repair. Arthroscopy 2016;32: 428-434. doi:10.1016/j.arthro.2015.08.021.

20. Walton JR, Murrell GA. A two-year clinical outcomes study of 400 patients, comparing open surgery and arthroscopy for rotator cuff repair. Bone Joint Res 2012;1: 210-217.

21. Brislin KJ, Field LD, Savoie FH 3rd. Complications after arthroscopic rotator cuff repair. Arthroscopy 2007;23: 124-128.

22. Berjano P, González BG, Olmedo JF, Perez-España LA, Munilla MG. Complications in arthroscopic shoulder surgery. Arthroscopy 1998; 14:785-788.

23. Curtis AS, Snyder SJ, Del Pizzo W, Friedman MJ, Ferkel RD, Karzel RP. Complications of shoulder arthroscopy. Arthroscopy 1992;8:395.

24. Huberty DP, Schoolfield JD, Brady PC, Vadala AP, Arrigoni P, Burkhart SS. Incidence and treatment of postoperative stiffness following arthroscopic rotator cuff repair. Arthroscopy 2009;25:880-890. doi:10.1016/j.arthro. 2009.01.018.

25. Muller D, Landsiedl F. Arthroscopy of the shoulder joint: A minimal invasive and armless procedure. Arthroscopy 2000;16:425.

26. Gallagher BP, Bishop ME, Tjoumakaris FP, Freedman KB. Early versus delayed rehabilitation following arthroscopic rotator cuff repair: A systematic review. Phys Sportsmed 2015;43:178-187. 
27. Wright RW, Baumgarten KM. Shoulder outcomes measures. J Am Acad Orthop Surg 2010;18:436-444.

28. Pollock RG, Flatow EL. The rotator cuff. Full-thickness tears. Mini-open repair. Orthop Clin North Am 1997;28:169-177.

29. Norberg FB, Field LD, Savoie FH 3rd. Repair of the rotator cuff. Mini-open and arthroscopic repairs. Clin Sports Med 2000; 19:77-99.

30. Ghodadra NS, Provencher MT, Verma NN, Wilk KE, Romeo AA. Open, mini-open, and all-arthroscopic rotator cuff repair surgery: indications and implications for rehabilitation. J Orthop Sports Phys Ther 2009;39:81-89.

31. Severud EL, Ruotolo C, Abbott DD, Nottage WM. All-arthroscopic versus mini-open rotator cuff repair: A long-term retrospective outcome comparison.
Arthroscopy 2003;19:234-238. doi:10.1053/jars.2003. 50036 [review].

32. Bishop J, Klepps S, Lo IK, Bird J, Gladstone JN, Flatow EL. Cuff integrity after arthroscopic versus open rotator cuff repair: A prospective study. J Shoulder Elbow Surg 2006;15: 290-299.

33. Heyer JH, Kuang X, Amdur RL, Pandarinath R. Identifiable risk factors for thirty-day complications following arthroscopic rotator cuff repair. Phys Sportsmed 2018;46: 56-60. doi:10.1080/00913847.2018.1388732.

34. Schairer WW, Nwachukwu BU, Fu MC, Warren RF. Risk factors for short-term complications after rotator cuff repair in the United States. Arthroscopy 2018;34: 1158-1163. doi:10.1016/j.arthro.2017.10.040. 